\title{
ANÁLISIS DE ACIERTOS Y BRECHAS EN LA PROMOCIÓN DE LA PARTICIPACIÓN CIUDADANA EN NIÑOS, NIÑAS Y JÓVENES DE LA CIUDAD DE MEDELLÍN. CASO PROYECTO SEMILLEROS INFANTILES
}

PARA LA PARTICIPACIÓN*

ANALYSIS OF SUCCESSES AND GAPS IN THE PROMOTION OF CITIZEN

PARTICIPATION IN CHILDREN AND YOUTH OF MEDELLÍN. CASE PROJECT CHILDREN

NURSERIES FOR CITIZEN PARTICIPATION

\section{EDINSON GABRIEL BRAND MONSALVE **}

\section{R E S U M E N}

Los semilleros infantiles para la participación desarrollados durante más de una década en Medellín son un proceso formativo para la generación de una ciudadanía activa; no obstante, aún no se han dimensionado sus resultados en la población beneficiaria. De aquí, el propósito del estudio fue evidenciar el nivel de cumplimiento de sus objetivos en la construcción de ciudadanía y en la transformación de conductas sociales y políticas en los beneficiarios. Se realizaron talleres grupales para la identificación logros, aciertos y brechas. Los resultados evidenciaron el fortalecimiento de las relaciones de convivencia, la construcción de redes sociales, el conocimiento y apropiación del territorio, a través de metodologías lúdicas, como el aporte más significativo en la construcción de ciudadanía.

PALABRAS CLAVE: participación, ciudadanía, juventud, niñez.
A B S T R A C T

The Children Nurseries for participation developed for more than a decade in Medellin, are a formative process for the generation of an active citizenship; however, their results had not yet been measured in the beneficiary population. Hence, the purpose of the study was to demonstrate the level of compliance with its objectives in the construction of citizenship and in the transformation of social and political behavior in the beneficiaries. Group workshops were held to identify achievements, successes and gaps. The results showed the strengthening of relations of coexistence, the construction of social networks, the knowledge and appropriation of the territory, through playful methodologies, are the most significant contribution in the construction of citizenship.

KEYWORDS: participation, citizenship, youth, childhood.

\footnotetext{
"Se presentan resultados que hacen parte de la investigación: "Evaluación de impactos de los semilleros infantiles para la participación ciudadana de Medellín, 2002 - 2013", concluida en el año 2016 y financiada por la Secretaría de Participación Ciudadana de la Alcaldía de Medellín.

** Magister en Desarrollo. Docente-Investigador Departamento de Sociología, Universidad de Antioquia, Medellín Colombia. edinson.brand@udea.edu.co
} 


\section{INTRODUCCIÓN}

Semilleros Infantiles para la participación es un proyecto adscrito a la Secretaría de Participación Ciudadana que viene ejecutándose hace más de 20 años, siendo actualmente una política pública (acuerdo 186 de 2013) que vincula niños y jóvenes de todas las comunas y corregimientos de la ciudad de Medellín. Este proyecto, al vincular a los habitantes de edades más tempranas al ejercicio de la ciudadanía se convierte en la antesala de la participación juvenil en la ciudad, la cual cuenta con diferentes programas para fortalecer la presencia de los jóvenes en escenarios de representación y toma de decisiones, como son PP Joven (Acuerdo 46 de 2010) que representa un espacio de fortalecimiento de la participación en Planeación Local y Presupuesto Participativo (Acuerdo 43 de 2007) en donde los jóvenes han adquirido una participación significativa, Consejo Municipal de Juventud ${ }^{1}$ (acuerdo 07 de 1994), Clubes juveni$\operatorname{les}^{2}$ (Acuerdo 083 de 2009) y Escuela Joven $^{3}$, entre otros.

\footnotetext{
Información en http://consejomunicipaldejuventud.blogspot.com/ 2 Información joven.com/que-es-clubes-juveniles
}

El proyecto Semilleros Infantiles para la participación tiene como propósito "promover una formación ciudadana que fomente la participación democrática de los niños, niñas y jóvenes en su territorio; desplegando un relevo generacional de los liderazgos zonales y locales" (Universidad de Antioquia y Alcadia de Medellín, 2013, p. 38), donde la concepción de ciudadanía admite diversas definiciones de acuerdo a su contexto y a los acontecimientos; para este caso específico se entiende “(...) como un concepto propio de la sociedad democrática, que garantiza el disfrute de derechos e implica la responsabilidad de deberes" (Gutiérrez y Morales, 2015, pág. 261) "Entender la ciudadanía como un concepto de doble dimensión, significa reconocer la importancia del ejercicio de la misma, resaltar la participación y la necesidad de buscar fórmulas que garanticen la convivencia, favoreciendo las normas y valores compartidos, así como el desarrollo de la conciencia colectiva" (Gutiérrez \& Morales, 2015, p. 262). Por ello, cabe señalar que los propósitos de creación de los semilleros infantiles, buscan la formación de una ciudadanía que entienda su rol dentro de su propio con-

\footnotetext{
3 Información en https://www.medellinjoven.com/buscamos-promotores-juveniles-para-escuela-joven-en-todas-las-zonas-de-la-ciudad-3753
} 
texto y realidad; que tenga una interacción consciente de los procesos que se llevan a cabo en la vida cotidiana.

Ahora bien, los acercamientos al fenómeno de la participación infantil y juvenil en América Latina han generado diferentes discusiones que se traducen en los retos que deben asumirse desde diferentes actores para gestionar las agendas públicas juveniles, que se desarrollan en escenarios caracterizados por la influencia de la tecnología y las visiones alternativas de participación. Entre estos retos el que mayor cantidad de trabajos agrupa tiene que ver con la necesidad trascender escenarios formales de participación hacia escenarios que incorporen otras dimensiones del ser niño y del ser joven, donde sus formas de expresión se presentan de manera alternativa a las estructuras institucionales, con códigos propios que representan sus intereses. Aquí es necesario emplear recursos prácticos que motiven a esta población a expandir sus capacidades y potencialidades en una participación ciudadana activa. (Carrasco, Carrasco y Guerra, 2014; Enríquez, 2015; Fernandez, 2014; Kriger y Daiban, 2011; Martuccelly, 2016; Reguillo, 2003).

Desde la perspectiva del Estado se encuentra la necesidad de orientar desde acciones que integren los servicios ofertados para la promoción de la participación infantil y juvenil, así como la consolidación de una política pública que defina las reglas que caracterizarán la acción de las instituciones públicas en favor de la integración de la población infantil y juvenil a los asuntos públicos, dando a esta población herramientas que los visibilicen con voz y voto para incidir en forma directa en los aspectos que les afecta.(Abad, 2002; Benedicto, 2016; Vélez, 2014).

Otro reto tiene que ver con el papel de la institución educativa, donde se requiere el desarrollo y consolidación de un clima escolar que estimule la participación, con fundamento en la convivencia la confianza y el respeto, que se apoye en diferentes espacios como la formación artística y que vele por garantizar igualdad en la calidad de la educación pública y privada.(Carrasco et al., 2014; Fernandeze, 2014; Martínez, Silva, Morandé, \& Canales, 2010; Ñáñez \& Castro, 2016).

A esto se suma la necesidad de sensibilizar y formar en participación y ciudadanía desde la niñez, promoviendo la participación infantil en escenarios que posibiliten la gestión para el cumplimiento de los derechos de los niños, lo cual conduzca a la modificación en las representaciones sociales que estos tienen acerca de la gestión pública 
y de la participación misma, fundamentando esta sensibilización y formación en la resiliencia y la aceptación activa de la diferencia. (Alvarado, Ospina, Luna, \& Camargo, 2006; Corvera, 2014; Gülgönen, 2016; Osorio, 1986).

En el marco de estas discusiones, se evidencia la necesidad de contar con investigaciones evaluativas que permitan comprender el estado actual de los procesos de formación y promoción de la participación infantil y juvenil, de cara a introducir mejoras que estén sincronizadas con la situación de esta población en el país y en América latina. Así, el propósito de la investigación fue reconocer los aspectos que como aciertos han facilitado el proceso de los Semilleros infantiles para la participación en Medellín, así como aquellos que se presentan como brechas que obstaculizan la materialización del escenario deseado por el proyecto en la construcción de ciudadanía.

\section{METODOLOGÍA}

Para el logro del objetivo propuesto se implementó la técnica de grupos de discusión para hacer análisis de aciertos y brechas respecto a los propósitos del proyecto. El punto de partida del trabajo realizado se fundamenta en la existencia de un escenario deseado representado por los propósitos del proyecto, los cuales se materializan mediante el objetivo central y los diferentes componentes del mismo, de donde se definen los elementos a evaluar. Esta parte del estudio buscó realizar un paralelo entre lo planeado y lo logrado, procurando una valoración a través del acuerdo colectivo entre los actores participantes de los procesos para dar cuenta de los aspectos que explican el logro o no de lo propuesto en el proyecto.

El trabajo grupal buscó responder las preguntas orientadoras ¿cuál fue el objetivo del proyecto al iniciar? y ¿cuáles fueron los logros obtenidos al finalizar la participación en el proyecto?. Estas preguntas se respondieron a partir de tres componentes: propósitos del proyecto según la percepción del participante, temas desarrollados más significativos y actividades realizadas que más le aportaron al logro de los propósitos y los temas. Estas preguntas se aplicaron sin dar información previa a los participantes, esto para reducir la posibilidad de sesgo en sus respuestas y reconocer lo que quedó instalado desde su experiencia. A partir de la reflexión colectiva de los participantes se identificaron los aspectos que estos reconocen como resultado logrado en cada componente, agrupándolos de acuerdo 
con las categorías establecidas (ver cuadro 1). De acuerdo a la cantidad de participantes que reconocen aspectos de logro en cada categoría se estableció la frecuencia porcentual de casos respecto al total de participantes. A partir de los aspectos reconocidos como logros por los participantes se identificaron aquellas situaciones o condiciones que los facilitaron (aciertos) y las que los dificultaron (brechas), siguiendo la misma metodología de agrupación de casos por categorías para establecer los porcentajes de casos en cada una respecto al total abordado.

La población abordada corresponde a 321 niños, niñas y jóvenes de 15 comunas $^{4}$ y 5 corregimientos de la ciudad que participaron en el proceso en una o más vigencias del mismo durante los años 2002 a $2013^{5}$, procurando que hubieran pasado por lo menos 2 años del egreso del programa, para valorar la experiencia en el mismo en el largo, mediano y corto plazo. Este período de tiempo se estableció con base a la información disponible en las bases de datos encontradas en el archivo de la Alcaldía de Medellín y organizaciones operadoras, verificando con un acercamiento previo la viabilidad del estudio de acuerdo la disponibilidad

\footnotetext{
${ }^{4}$ La única comuna que no se pudo integrar al estudio fue la 14 Poblado, dado que no se ubicaron participantes en el período definido.
}

de personas para participar en los encuentros.

En total se realizaron 27 talleres que vincularon un máximo de 12 personas en cada uno. En estos espacios se propició la interacción de los participantes para lograr acuerdos frente a qué aspectos (situaciones, acciones, condiciones, comportamientos) alejaron el proyecto del propósito inicial (brechas) y cuales lo acercaron (aciertos), consignando los resultados en una matriz. A cada uno de los enunciados concertados por los participantes se les asignó un código por frecuencia de casos, permitiendo identificar la participación de cada aspecto con base al total de la población.

\section{RESULTADOS}

Los resultados dan cuenta de dos componentes abordados en esta parte de la investigación: en primer lugar, los elementos formales del proyecto que presentan un mayor reconocimiento por los participantes, de acuerdo con la importancia que significaron en la experiencia dentro del proceso formativo. En segundo lugar, asociados a estos elementos, los aspectos que más les facilitaron alcanzar el escenario deseado por el proyecto, y aquellos

\footnotetext{
${ }^{5}$ El proyecto se ejecuta con una vigencia anual, habiéndose mantenido ininterrumpido por 25 años.
} 
que más los limitaron, en otras palabras, los aciertos y las brechas del proceso formativo respectivamente. Los resultados se presentan a través de frecuencias porcentuales resultantes de la agrupación de las respuestas de los participantes en categorías emergentes, discriminadas por área urbana y área rural.

\subsection{Elementos formales de mayor reconocimiento}

Los elementos formales del programa corresponden a aquellos que fueron establecidos en su diseño, identificando el alcance esperado con su implementación, a saber: el propósito del programa, que se traduce en dimensiones y categorías. Allí se reconocen los contenidos que se procuraron dejar instalados en la experiencia de los participantes. Para su valoración, en esta parte de la investigación se indagó a los niños, niñas y jóvenes por los propósitos del proyecto que reconocen, los temas desarrollados a lo largo del proceso formativo que representaron el aporte del proyecto a sus experiencias y las actividades a través de las cuales se desarrollaron los temas.

\subsubsection{Propósitos del proyecto}

En este primer componente, de carácter general, se buscó comprender el marco de comprensión en el cual los participantes ubican los logros obtenidos en el proyecto. Como punto de partida se tiene que para el proyecto la formación en participación ciudadana propuesta va más allá de la participación política o en escenarios políticopartidistas, aunque los incluye, es decir, se entiende la ciudadanía como el ejercicio de derechos y deberes, pero vinculado a la integración con el territorio y la comunidad a partir del trabajo colectivo e individual ligado al contexto y al lenguaje e intereses de la población infantil y juvenil.

En este marco, la información obtenida en los encuentros realizados en las comunas y corregimientos de la ciudad muestra que las categorías de mayor reconocimiento frente al propósito del programa son diferentes en lo urbano y en lo rural, de donde es claro un primer asunto y es que la forma de percibir y apropiarse de la ciudadanía, a través de conocimientos, valores, normas, etc., tiene una relación muy fuerte con el área del individuo y de los grupos. Nótese en el gráfico 2 que la población infantil y joven del área urbana tiene un mayor reconocimiento por la convivencia y por la diversión como propósito, seguidas del reconocimiento del territorio y la comunidad $(54.8 \%, 55.2 \%$ y $32.9 \%$ de los casos abordados respectivamente), en tanto que la población del 
área rural lo tiene por la convivencia (38.6\%), el aprendizaje de valores (38.6\%), el trabajo en equipo (37.3\%) y la formación política (33.3\%), seguidas por el conocimiento del territorio y la comunidad (28\%).

Una observación importante en este punto es entender que estas categorías no están aisladas unas de otras; es decir, el hecho que una categoría gane más reconocimiento no significa que otras lo pierdan, por lo que debe hacerse una lectura sistémica, esto es, aquí se está evidenciando cuáles son las categorías, qué como propósito del proyecto para los participantes, está permitiendo la dinamización de las otras.

De aquí se evidencia que en lo urbano se dio una mayor tendencia entre los participantes a acoger dos categorías de manera predominante, como las que mayor significación le dan al propósito del programa, en tanto que en lo rural se reconocen cuatro, es decir, al interior del área rural se encuentra una diferencia entre la población abordada, respecto a las categorías que representan mejor el propósito del proyecto. De aquí puede decirse que en lo urbano es más significativo para los niños, niñas y jóvenes entender los Semilleros de participación como un espacio de construcción de vínculos interpersonales para apren- der a convivir con otros, a partir de actividades lúdicas y recreativas como juegos, dibujo, salidas, entre otras, siendo la diversión el dispositivo a través del cual se capta la atención e interés la población para canalizar los contenidos y el desarrollo de habilidades para la participación ciudadana fundamentada en la convivencia.

En el área rural, la convivencia también es una categoría representativa del propósito del proyecto, más no está acompañada por la diversión, sino por asuntos más concretos como los valores y el trabajo en equipo, lo cual no quiere decir que estos niños, niñas y jóvenes no se diviertan en el proceso, sino que para ellos lo que está mediando la participación no es la diversión, sino los valores y el trabajo en equipo, acompañado de la formación política. En ambos casos, es importante hacer relevante que la participación se está dinamizando a través de otros aspectos que involucran más lo colectivo, el comportamiento grupal, lo cual está acompañado del conocimiento del territorio y la comunidad, indicado la construcción de una visión de participación y ciudadanía vinculada al otro y al territorio, siendo un logro significativo en la ciudad teniendo en cuenta la necesidad de vincular al niño y al joven con los asuntos de su comunidad, como un aspecto 
fundante del liderazgo para la participación ciudadana.

Un aspecto a tener en cuenta en el análisis de porque no hay categorías que agrupen una mayoría de la población, haciéndolas más representativas como propósito del programa, es que la operación administrativa y pedagógica se ha dado por diversas entidades en el período de tiempo investigado, encontrando que quienes orientaron y definieron los lineamientos del proyecto no siempre se articularon a los precedentes, por lo que cada entidad pudo haber hecho énfasis en categorías diferentes para dinamizar y canalizar la participación en la población intervenida.

\section{FIGURA 1. PROPÓSITOS DEL PROGRAMA RECONOCIDOS POR LOS PARTICIPANTES ${ }^{6}$}

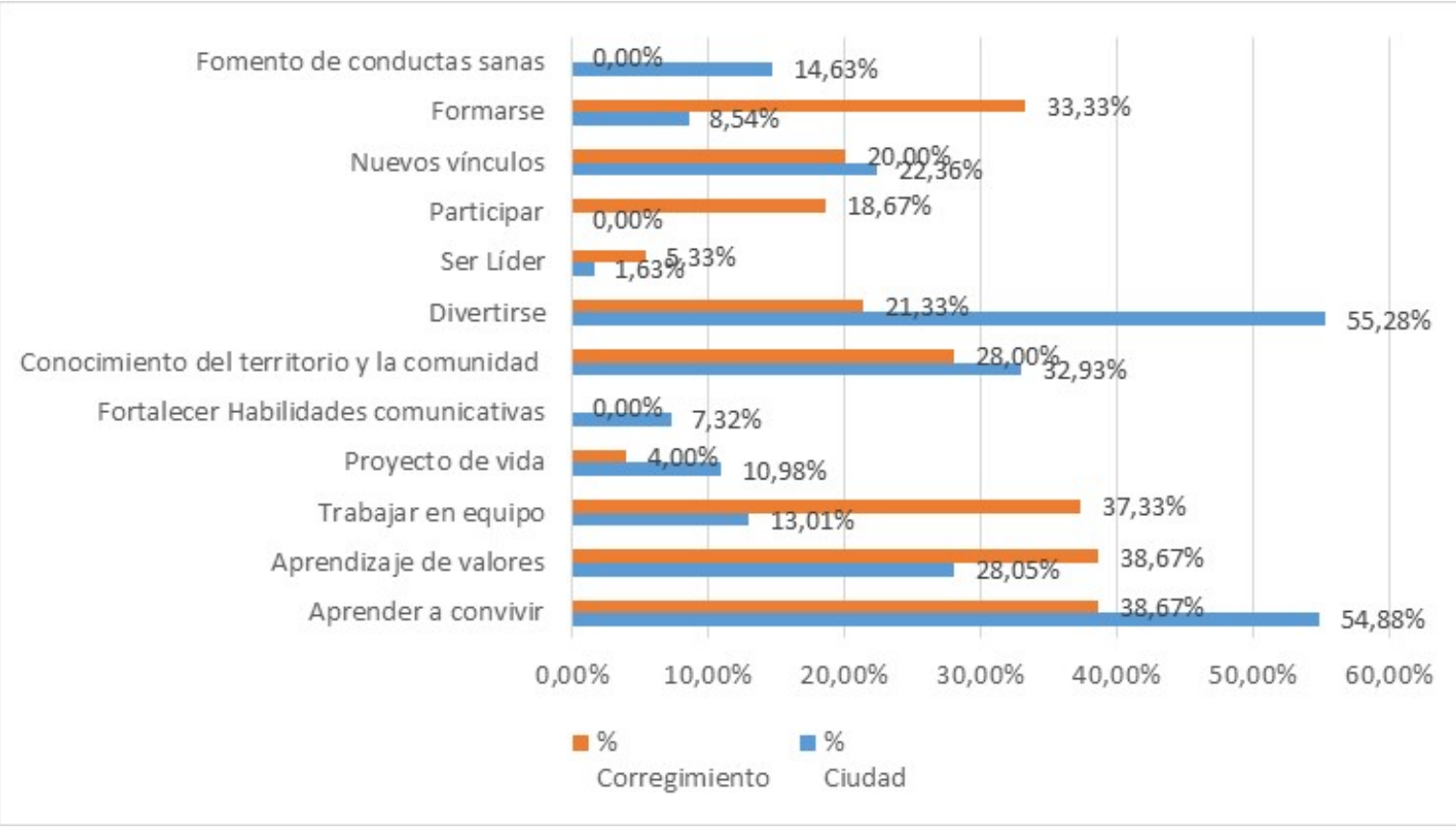

Fuente: elaboración propia

Otro aspecto importante a resaltar es que

el propósito de la participación haya sido reconocido solo por el $18,67 \%$ de los entrevistados en los corregimientos y en las comunas no reporte ninguna cifra. Además, que la variable li- derazgo mantenga la misma tendencia (solo figura en lo urbano con 1,63\% y en rural con el 5,33\%). Esta situación puede estar señalando que no hay suficiente claridad a la hora de trasmitir el mensaje sobre las intencionales

${ }^{6}$ Para todos los gráficos presentados en el texto, en cada ítem el porcentaje representa la proporción de casos frente al total de la población. 
del proyecto en la población beneficiaria, falencias que pueden tener su raíz en la falta de unidad de criterios, generados por las condiciones administrativas de cambios constantes en los operados y dinamizadores, sin que medien empalmes en los diferentes componentes del proyecto, toda vez que son estos los encargados de orientar los contenidos sociales, políticos y culturales de los diferentes grupos de semilleros en las comunas y corregimientos.

\subsubsection{Temas trabajados en el proyecto}

Vinculados a estos propósitos, los temas que mayor relevancia tuvieron para la población, de acuerdo con su experiencia en el proceso, corresponden en el área rural con la vinculación al territorio y las condiciones del territorio (48\% y $46.6 \%$ respectivamente, ver gráfico 2). Entendiendo con esto que, en el primer caso, apropiarse del territorio fue uno de los temas que más marcó a los niños, niñas y jóvenes participantes, los cuales en muchos casos expresaban su satisfacción de sentir el vecindario como propio, especialmente aquellos que vivían en entornos con presencia de enfrentamientos de grupos armados y fronteras invisibles. El segundo tema, se torna importante dado que es el paso necesario para que el primero logre un aporte en la vida de los participantes, dado que para apropiarse del territorio es necesario reconocer sus condiciones, registrando que los recorridos por los barrios y las comunas, así como las actividades que involucraban a la comunidad, marcaron significativamente el paso de los participantes por el proyecto.

De aquí se evidencia que estos temas fueron trabajados en el marco de la convivencia, el reconocimiento de valores y el trabajo en equipo, acompañado del conocimiento por el territorio y la comunidad. Nótese que los temas tienen un comportamiento diferente al elemento anterior, respecto de la frecuencia de casos; es decir, se evidencia que los porcentajes de casos para quienes estos dos temas son relevantes corresponde a casi la mitad de la población en cada tema, apareciendo luego otra serie de temas que se vuelven secundarios o complementarios a estos principales, de donde se nota que si bien los propósitos del programa están un poco más dispersos, en el desarrollo de contenidos se logra mayor unificación de la percepción. Lo que sugiere que, en el caso del área rural, la formación y promoción de la participación ciudadana en la población intervenida se tornó significativa al vincularse con el territorio, en un escenario que oferta esta participa- 
ción como un proceso asociado a la generación de relaciones de convivencia y trabajo en equipo, sustentado en valores.

En el área urbana se observa un compartimiento también diferente al observado en el elemento anterior de los propósitos del programa, respecto de la frecuencia porcentual de casos asociados, registrando tres temas que tuvieron un mayor reconocimiento entre los participantes, pero que no alcanzaron a agrupar más de la mitad de estos. A saber, el reconocimiento de los conflictos $(36,59 \%)$, vinculación al territorio (34,15\%), y la generación y consolidación de vínculos personales (30,49\%); reflejando que en el caso de los temas se da una orientación diferenciada en su apropiación por parte de la población.

Ahora bien, teniendo en cuenta los propósitos reconocidos, se puede establecer que hay un coherencia de los temas que fueron importantes en el marco de estos, dado que los mismos están orientados a alcanzar el establecimiento de relaciones de convivencia, toda vez que el reconocimiento de los conflictos es fundamental a este propósito, así como la generación de vínculos entre los participantes, lo cual se hace significativo en el marco del territorio, coincidiendo en ello con la población del área rural, es decir, en el conocimiento y la vinculación al territorio; por lo que puede decirse que este es el aspecto más transversal a la formación y promoción de la participación en la ciudad. Nótese además que los temas que están en un segundo nivel, con frecuencia cercanas, de importancia para la población urbana complementan a estos primeros: mecanismos de intervención para dirimir/administrar conflictos (22,76\%), condiciones del territorio (multisectorial) con $(21,95 \%)$ y relacionamiento en general con la comunidad y las instituciones (21,14\%).

Sobre estos dos elementos trabajados se evidencia que para la población rural hay un mayor acuerdo frente a los temas que frente a los propósitos del proyecto, en tanto que para la población urbana ocurre lo contrario, lo que representa diferencias importantes que marcan el nivel de impacto que el proyecto ha tenido y tiene en cada tipo de población. 


\section{FIGURA 2. TEMAS DEL PROGRAMA RECONOCIDOS POR LOS PARTICIPANTES}

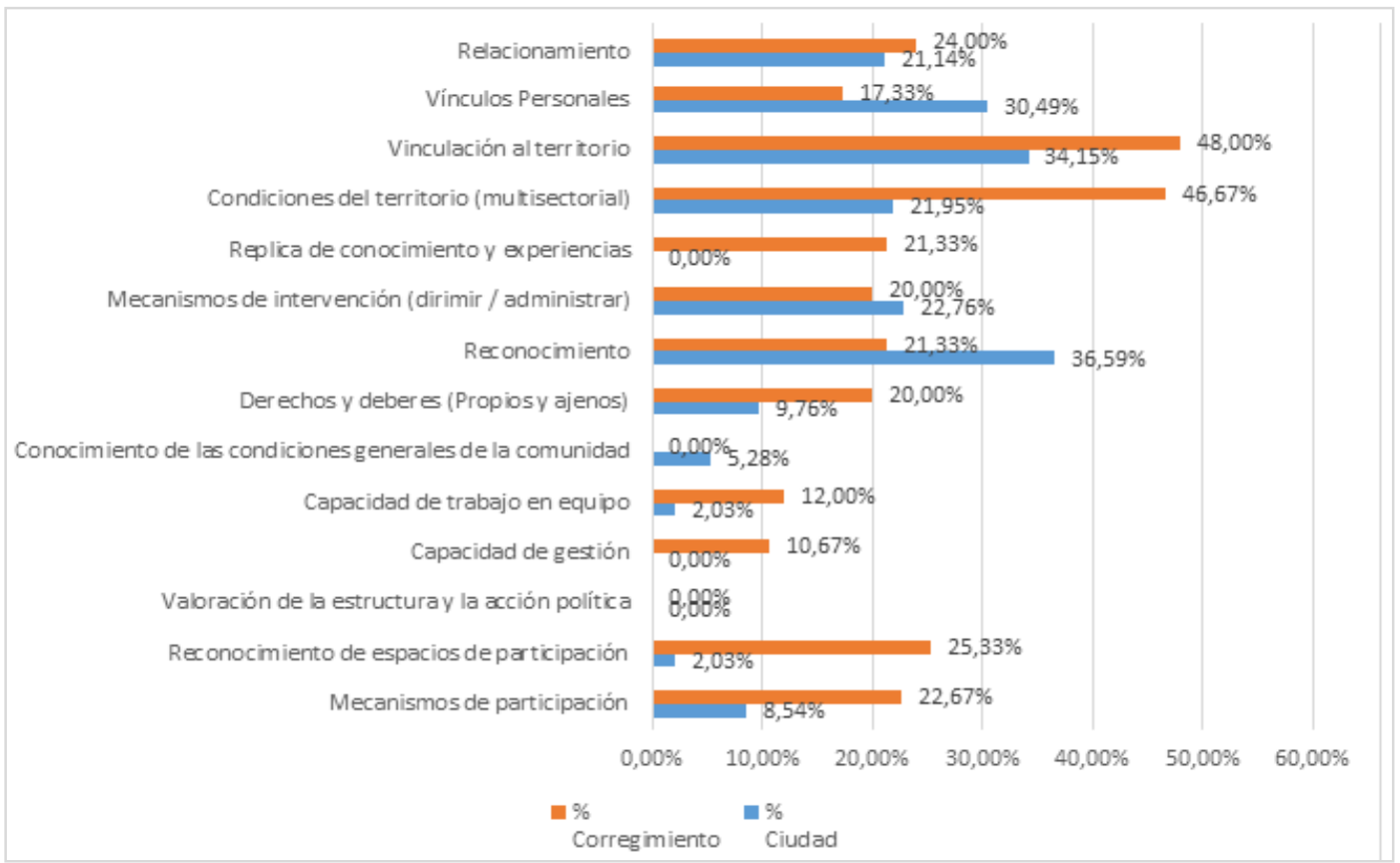

Fuente: elaboración propia

A partir de los aspectos reconocidos como temas, con base a la experiencia de los participantes, se encuentra la siguiente matriz de resultados respecto a la estructura operacional del proyecto. Es importante aclarar que en la dinámica del proyecto, en sus vigencias anuales, no se establecieron indicadores de impacto que permitieran contrastar los resultados obtenidos, por lo que en esta evaluación se reconoce lo logrado haciendo énfasis en aquellos aspectos que fueron obtenidos solo a través de la participación en el proyecto, convirtiéndose a su vez en la línea base para las vigencias posteriores.

\section{CUADRO 1. MATRIZ DE EVALUACIÓN DEL PROYECTO}

\begin{tabular}{|c|c|c|c|c|}
\hline \multicolumn{3}{|c|}{ ESCENARIO DISEÑADO } & \multicolumn{2}{|c|}{ LOGROS* } \\
\hline $\begin{array}{l}\text { DIMENSIONE } \\
\text { S }\end{array}$ & CATEGORIAS & VARIABLES & URBANO & RURAL \\
\hline \multirow{6}{*}{$\begin{array}{l}\text { Competencia } \\
\text { s Cívicas }\end{array}$} & $\begin{array}{l}\text { Conocimientos en } \\
\text { participación }\end{array}$ & $\begin{array}{l}\text { Conocimiento de mecanismos de } \\
\text { participación }\end{array}$ & $8,94 \%$ & $46,67 \%$ \\
\hline & \multirow{2}{*}{$\begin{array}{l}\text { Habilidades de } \\
\text { participación en espacios } \\
\text { colectivos }\end{array}$} & $\begin{array}{l}\text { Reconocimiento de espacios de } \\
\text { participación }\end{array}$ & $2,03 \%$ & $52,00 \%$ \\
\hline & & $\begin{array}{l}\text { Valoración de la estructura y la } \\
\text { acción política }\end{array}$ & $0,00 \%$ & $37,33 \%$ \\
\hline & \multirow{2}{*}{ Habilidades de liderazgo } & Capacidad de gestión & $0,00 \%$ & $21,33 \%$ \\
\hline & & Capacidad de trabajo en equipo & $1,63 \%$ & $21,33 \%$ \\
\hline & $\begin{array}{l}\text { Fomento de la } \\
\text { participación }\end{array}$ & $\begin{array}{l}\text { Conocimiento de las condiciones } \\
\text { generales de la comunidad }\end{array}$ & $4,07 \%$ & $0,00 \%$ \\
\hline \multirow[t]{2}{*}{$\begin{array}{l}\text { Participación } \\
\text { Activa }\end{array}$} & Reconocimiento & $\begin{array}{l}\text { Derechos y deberes (Propios y } \\
\text { ajenos) }\end{array}$ & $9,35 \%$ & $44,00 \%$ \\
\hline & & Reconocimiento & $30,49 \%$ & $38,67 \%$ \\
\hline
\end{tabular}




\begin{tabular}{|l|l|l|c|c|}
\hline $\begin{array}{l}\text { Relaciones de } \\
\text { convivencia }\end{array}$ & $\begin{array}{l}\text { Manejo adecuado de } \\
\text { conflictos }\end{array}$ & $\begin{array}{l}\text { Mecanismos de intervención } \\
\text { (dirimir / administrar) }\end{array}$ & $27,24 \%$ & $37,33 \%$ \\
\hline \multirow{4}{*}{$\begin{array}{l}\text { Capacidad de } \\
\text { Gestión }\end{array}$} & $\begin{array}{l}\text { Intercambio de } \\
\text { conocimiento }\end{array}$ & $\begin{array}{l}\text { Replica de conocimientos y } \\
\text { experiencias }\end{array}$ & $0,00 \%$ & $38,67 \%$ \\
\cline { 2 - 5 } & $\begin{array}{l}\text { Conocimiento del } \\
\text { territorio }\end{array}$ & $\begin{array}{l}\text { Condiciones del territorio } \\
\text { (multisectorial) }\end{array}$ & $23,58 \%$ & $76,00 \%$ \\
\cline { 2 - 5 } & Vinculación al territorio & $32,93 \%$ & $69,33 \%$ \\
\cline { 2 - 5 } & Construcción de redes & Vínculos Personales & $30,08 \%$ & $33,33 \%$ \\
\cline { 2 - 5 } & Relacionamiento & $20,73 \%$ & $48,00 \%$ \\
\hline
\end{tabular}

Fuente: Elaboración propia.

\subsubsection{Actividades}

De manera complementaria a los logros identificados, en un tercer componente referido a las actividades que se tornaron más significativas para la población en el desarrollo de los temas puede decirse que en general las metodologías adoptadas han sido acertadas, de acuerdo con la experiencia de los niños, niñas y jóvenes abordados, encontrando un mayor acuerdo entre el área rural y la urbana. Se observa que en ambas poblaciones hay una alta valoración por las actividades lúdicas y pedagógicas y por las salidas y los recorridos realizados dentro y fuera de su territorio.

Teniendo las actividades lúdico-pedagógicas con una mayor valoración en lo urbano (66.6\% frente al 52\% en lo rural) y las salidas y recorridos con mayor valoración en lo rural (66.6\% frente al $40.2 \%$ en lo urbano). Esto se debe a que los niños, niñas y jóvenes del área rural salen menos de sus ve- cindarios, por la lejanía del centro urbano, y además porque sus actividades cotidianas se desenvuelven en torno a la vereda o en el centro poblado del corregimiento y por tanto son más ajenos a las dinámicas de la ciudad, por lo que este tipo de actividades resultaron muy novedosas y aportantes. En tanto que en lo urbano hay una mayor interacción con diferentes espacios de la ciudad, por lo que, si bien hay una valoración significativa de las salidas y recorridos, las actividades desarrolladas en el barrio a través del enfoque lúdico-pedagógico ocupan un primer lugar.

Un aspecto importante es que los talleres reflexivos aparecen relevantes, casi en igual proporción, tanto en lo urbano como en lo rural (42.6\% y $44 \%$ respectivamente), de donde es claro que si bien hay una valoración de lo lúdicopedagógico, hay un complemento con lo reflexivo para el abordaje de los temas trabajados en la promoción de la participación ciudadana. 


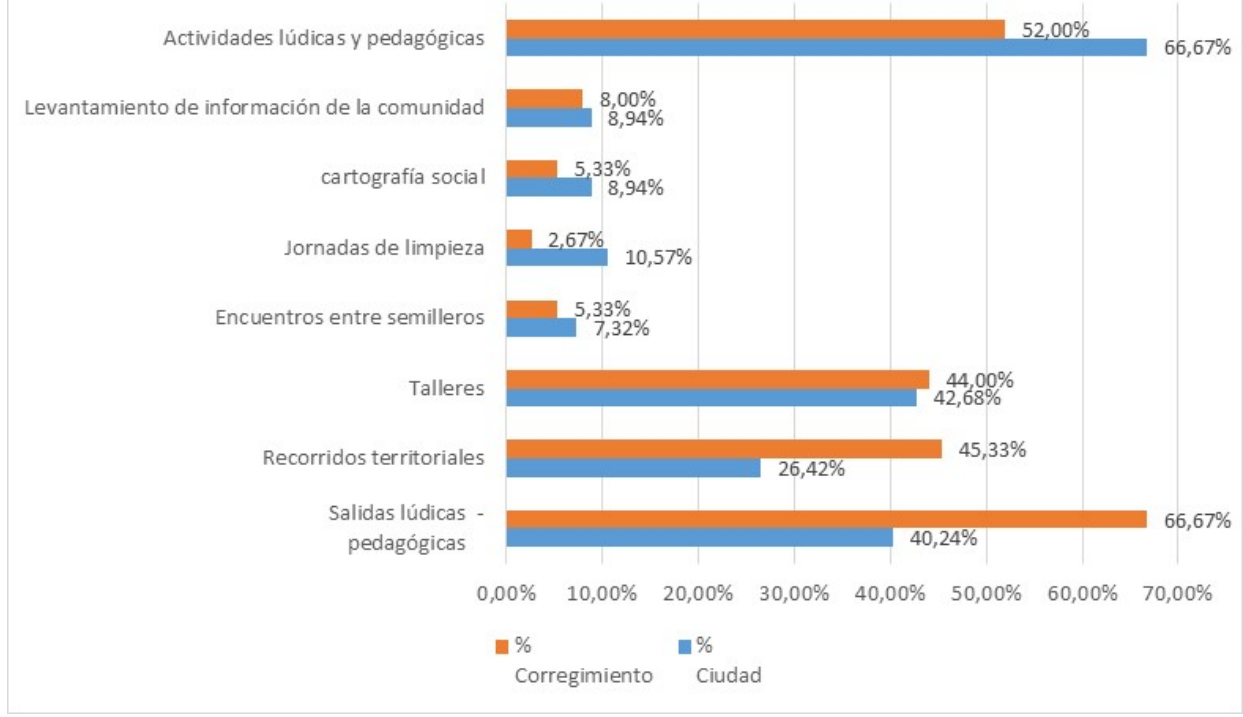

Fuente: Elaboración propia.

\subsection{Aciertos y Brechas del proyecto}

Frente a los aspectos reconocidos como temas que dan cuenta de los logros del proyecto con la población de niños, niñas y jóvenes participantes (Cuadro 1), el segundo componente abordado consistió en reconocer que aspectos facilitaron alcanzarlos y cuales obstaculizaron o fueron impedimentos, presentándose como grietas para lograr que los resultados fueran mayores. A los primeros se les denominó aciertos y a los segundos brechas. Los resultados se presentan a continuación a partir de la agrupación de la información aportada por los 321 participantes producto de la reflexión grupal de donde se derivaron acuerdos frente a estos aspectos, estableciendo posteriormente frases o palabras que representan categorías en las cuales se agrupan las diferentes percepciones con base a la frecuencia de repetición o coincidencia entre los participantes, estableciendo porcentajes de acuerdo a la proporción de casos observados para cada categoría respecto al total de casos abordados.

\subsubsection{Análisis de aciertos}

Igual que en el reconocimiento de los elementos formales del proyecto, los aciertos presentan diferencias entre el área urbana y el área rural, lo cual se puede entender en razón que las condiciones generales en cada territorio son muy diferentes, por lo que los resultados también se presentarán de manera diferenciada. 
En el área urbana se reconocen, casi en igual proporción de casos, cuatro situaciones que se presentaron como aciertos para alcanzar los resultados presentados: el relacionamiento $(35,7 \%)$, la metodología (34.5\%), las condiciones logísticas $(34,1 \%)$ y las condiciones del dinamizador (34.1\%). Se observa que el relacionamiento, a la vez que es un logro, se torna en una situación que ha favorecido alcanzar los otros logros, lo cual indica que las redes son un aspecto fundamental en la participación ciudadana, superando la concepción basada solo en el desarrollo de habilidades en el individuo, asumiendo la importancia de gestionar tales habilidades en el marco de la estructura de sus relaciones. Complementariamente se reconocen situaciones que tienen que ver con la organización del proyecto respecto a la metodología utilizada, que confirma lo encontrado en los propósitos del programa y en las actividades desarrolladas, siendo que la orientación lúdicopedagógica ha sido un elemento de gran valor para la población en razón de permitirles acercarse a temas tan complejos como la participación ciudadana desde un espacio que se les presenta más cercano a su condición de niños y jóvenes. Por último se tienen las condiciones logísticas y del dinamizador, en donde hay un claro reconocimiento a los recursos físicos y humanos dispuestos para el desarrollo de las actividades y el acompañamiento de los niños, dado que los dinamizadores, que son las personas que desarrollan las actividades con los niños, son en la mayoría de casos egresados del programa, es decir, personas formadas por el mismo programa, lo cual permite una cercanía con los participantes que redunda en vínculos fuertes entre el dinamizador y cada uno de los niños, niñas y jóvenes sobre la base de tener la misma experiencia, lo que torna a los dinamizadores en un referente para la población.

De otro lado, en el área rural se encuentra que la situación que se presenta como un claro acierto en el proceso corresponde al relacionamiento (42.6\%), lo que se explica a partir de la cercanía y la interacción que el programa le permitió a la población participante, en razón que en su cotidianidad los niños, niñas y jóvenes están más concentrados en actividades hogareñas cuando no están en la institución educativa. En este marco poder acercarse a la participación a partir del reconocimiento de otros pares con los mismos intereses, mediante actividades grupales, es un factor que facilita la apropiación de los contenidos trabajados. Sumándose a ello, se tiene que estas actividades grupales son cercanas a su lenguaje e intereses, 
toda vez que tienen una orientación lúdico-pedagógica, siendo este un segundo acierto significativo para la población (26.6\%), es decir, se acercó a los niños, niñas y jóvenes la oferta sobre la participación a partir de actividades que se basan en la diversión, evitando escenarios formales. De hecho, los temas que menos recordación tienen entre los niños corresponde a aquellos que son más técnicos como los mecanismos de participación, dado que son contenidos muy específicos que permiten pocas opciones de ser apropiados de manera práctica, en tanto que temas asociados a comprender e integrarse a los asuntos del territorio, la importancia de la convivencia, el reconocimiento del otro, etc., son aspectos que se tornan más cercanos a la vida cotidiana y práctica.

Otro acierto apreciado en mayor medida por los entrevistados en lo urbano corresponde con lo logístico (34.15\%), en contraste con los corregimientos donde solo lo mencionaron el 1,33\% de los participantes. Esto indica que la adecuada condición locativa de los lugares donde se desarrollaron las actividades del semillero fueron muy significativas y por tanto mayormente valoradas en lo urbano. Este dato podría explicarse en consideración que en el área urbana hay una mayor disponibilidad de espacios para el desarrollo de este tipo de proyectos, en tanto que en la rural es menor.

\section{FIGURA 4. ACIERTOS IDENTIFICADOS EN LA EXPERIENCIA DE LOS PARTICIPANTES}

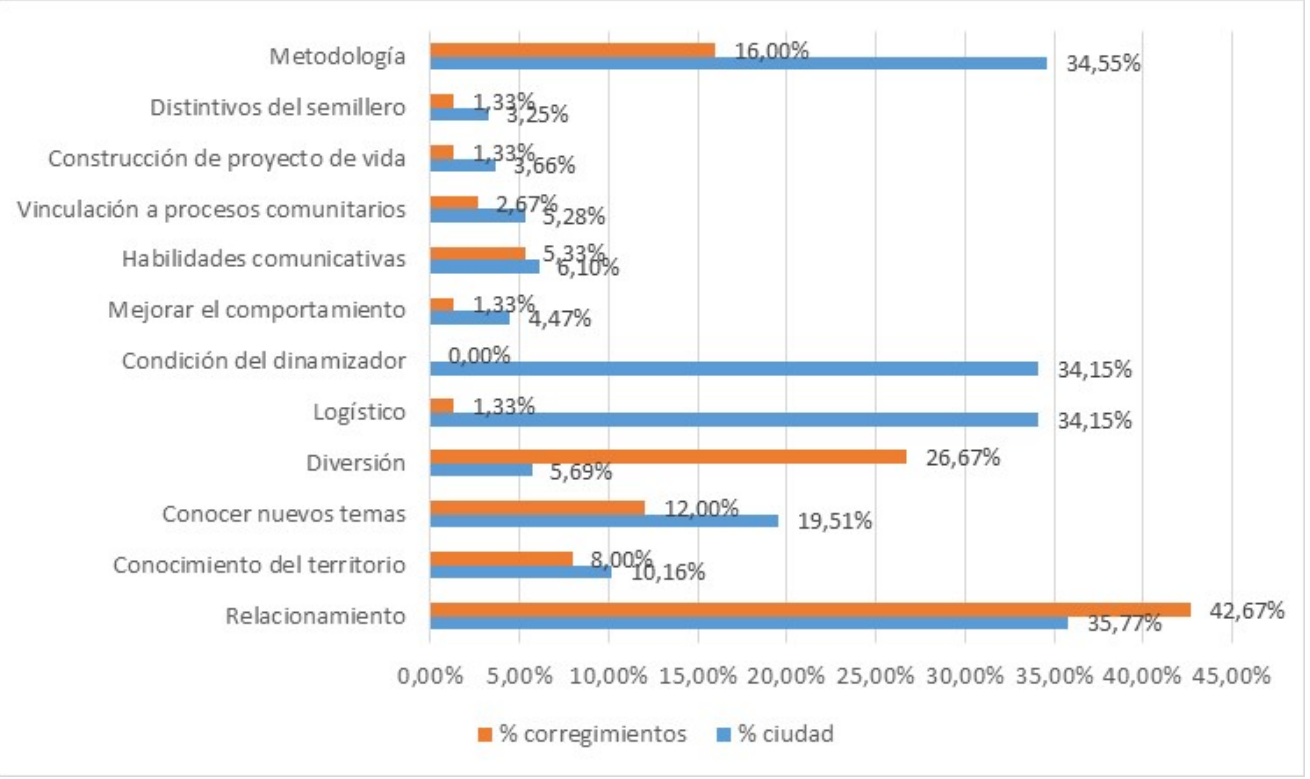

Fuente: elaboración propia. 
La metodología de trabajo utilizada por los dinamizadores en los encuentros también fue un acierto significativo para los entrevistados del área urbana, representando el $34,15 \%$ y en menor medida para los corregimientos con 16,00\%. Del mismo modo, en la variable condición de dinamizador, en lo urbano también presentó una cifra notable con el $34,15 \%$, pero en los corregimientos no reportó ningún dato, por lo que puede inferirse que en el primer caso se produjo una mayor identificación con la personalidad del dinamizador y esto aportó a la permanencia del grupo.

Frente a los aspectos que se presentan como aciertos es importante hacer algunas observaciones frente a aquellos que presentan contrastes significativos entre el área urbana y la rural, como es el caso de la condición del dinamizador y lo logístico, pues nótese que solo son vistos como aciertos en al área urbana. Esta situación puede explicarse en razón que en el centro urbano se cuenta con una mayor infraestructura disponible, así como equipamiento colectivo para el desarrollo de múltiples actividades, en tanto que en lo rural hay menos recursos físicos, teniendo que el espacio disponible para el trabajo con la población es fundamentalmente la sede la Junta de Acción Comunal.
Respecto al dinamizador, es claro que en la población rural esta figura no logra ser reconocida como un referente, tal como ocurre en lo urbano, sin tener clara la razón de ello con la información recogida en esta investigación. De igual forma se encuentra la situación asociada a la diversión, teniendo que en lo rural se presenta como un acierto con una proporción importante de casos (26.6\%), frente a lo urbano que se reconoce en pocos casos (5.6\%). Esta situación se explica en función que para la población urbana la diversión es una condición propia al proyecto, por lo que tiene un nivel más de propósito y por tanto no es un acierto sino una característica del programa. En contraste, para la población rural la diversión es un aspecto de las actividades desarrolladas que les permitió abordar los temas formativos, donde el aprendizaje y la formación tienen realmente más el lugar de propósito, como se vio en ese elemento.

Finalmente es relevante ver que emergieron algunos casos, tanto en lo urbano (5.2\%) como en lo rural (2.6\%), en los que se valora la vinculación a procesos comunitarios como un acierto para alcanzar los logros que fueron obtenidos en el proyecto, de donde es claro que este deberá ser un aspecto por fortalecer, toda vez que se torna en una estrategia directa para 
promover la participación de la población a través de su inclusión en los asuntos de sus comunidades.

\subsubsection{Análisis de brechas}

De otro lado, se indagó a la población entrevistada por las situaciones o condiciones que obstaculizaron el proceso y limitaron la posibilidad de haber obtenido mejores resultados, respecto a los presentados, identificando los aspectos en los cuales es necesario introducir mejoras en el proyecto para procurar un mayor impacto en las vigencias futuras del mismo. $\mathrm{Al}$ respecto se tiene una primera observación y es que no se registraron aspectos con altas frecuencias de ocurrencia, es decir, que agruparan un número considerable de respuestas, lo que indica que en general el programa se ha desarrollado con obstáculos que han limitado el proceso en un bajo nivel.

Teniendo en cuenta estas dos observaciones, y con base a la información del gráfico 5 , se encuentra en el área urbana que el aspecto que representó la brecha más significativa, aunque con un porcentaje bajo, corresponde a las condiciones logísticas (22.7\%), esto se da para algunos casos en los que los entrevistados expusieron que para varios encuentros no contaron con un lugar fijo para realizar las actividades del semillero, razón por la cual, debían ubicarse en lugares públicos que impedían la concentración o reunirse en las viviendas de los dinamizadores o participantes, situación que generó algunos inconvenientes para la participación. Aquí debe tenerse en cuenta que las condiciones logísticas fueron identificadas por una proporción mayor de casos (34.1\%) como una oportunidad, lo que indica que en una parte de las comunas de la ciudad se tuvo una disposición de recursos logísticos suficientes, mientras que en otras no, especialmente en lo relacionado con los espacios de encuentro.

Una segunda brecha, corresponde al conflicto entre los participantes (17.4\%), esto es, las peleas entre los mismos beneficiarios, teniendo en algunos casos agresiones físicas, siendo una situación que afectó el normal desarrollo de las actividades y por tanto el logro de estas mismas se vio limitado. Con base a esta brecha se entiende la brecha de baja participación, toda vez que como producto de los conflictos algunos niños, niñas y jóvenes optaban por retirarse del proyecto, aunque es una brecha que poco afectó el proceso, siendo identificado por el $4 \%$ del total de los entrevistados.

Finalmente podría mencionarse como una tercera brecha importante, aunque con un porcentaje de casos mucho menor, el cambio de dinamizador 
(11.3\%). Esta situación se dio en algunas comunas, en las cuales por razones administrativas o personales se cambió la persona dinamizadora, lo que afectó negativamente el proceso con la población dado el nivel de confianza logrado en el relacionamiento entre ambas partes, por lo que construir nuevas relaciones significó retrocesos en las actividades y temáticas, afectando además la participación de los niños, niñas y jóvenes. Aquí es importante tener en cuenta que la condición del dinamizador fue valorada como una oportunidad por una proporción significativa de respuestas (34.1\%), lo que hace que cualquier cambio en este aspecto impacte significativamente la experiencia de la población.

Respecto al área rural, se encuentra que la mayor brecha que reconocen los participantes corresponde a la baja participación (28\%), esto es, la baja disponibilidad en algunos asistentes para desarrollar los ejercicios propuestos por los dinamizadores, encontrado alguna resistencia en los asistentes y en otros casos debilidad en habilidades para la socialización y el relacionamiento. También se encuentra que otros aspectos influyeron en esta baja participación como la segunda brecha registrada respecto a la logística deficiente (22.6\%), que como ya se mencionó, tiene que ver con la baja disponibilidad de espacios para la realización de encuentros y de diferentes actividades lúdico-recreativas, lo que limitaba las posibilidades de realizar ejercicios a campo abierto o actividades deportivas, entre otros. En tercer lugar, se tiene una brecha asociada a las dificultades para asistir (21.3\%). Encontrando que en varios casos esto se dio por la lejanía al lugar de encuentro, pues, aunque el proyecto les cubría los costos de desplazamiento, se presentaban dificultades de otra índole como el cumplimento de tareas o la imposibilidad de encontrar un acompañante para el desplazamiento, generando una presencia intermitente que afectó en algunos corregimientos el normal desarrollo de las actividades y por tanto el impacto de estas. 


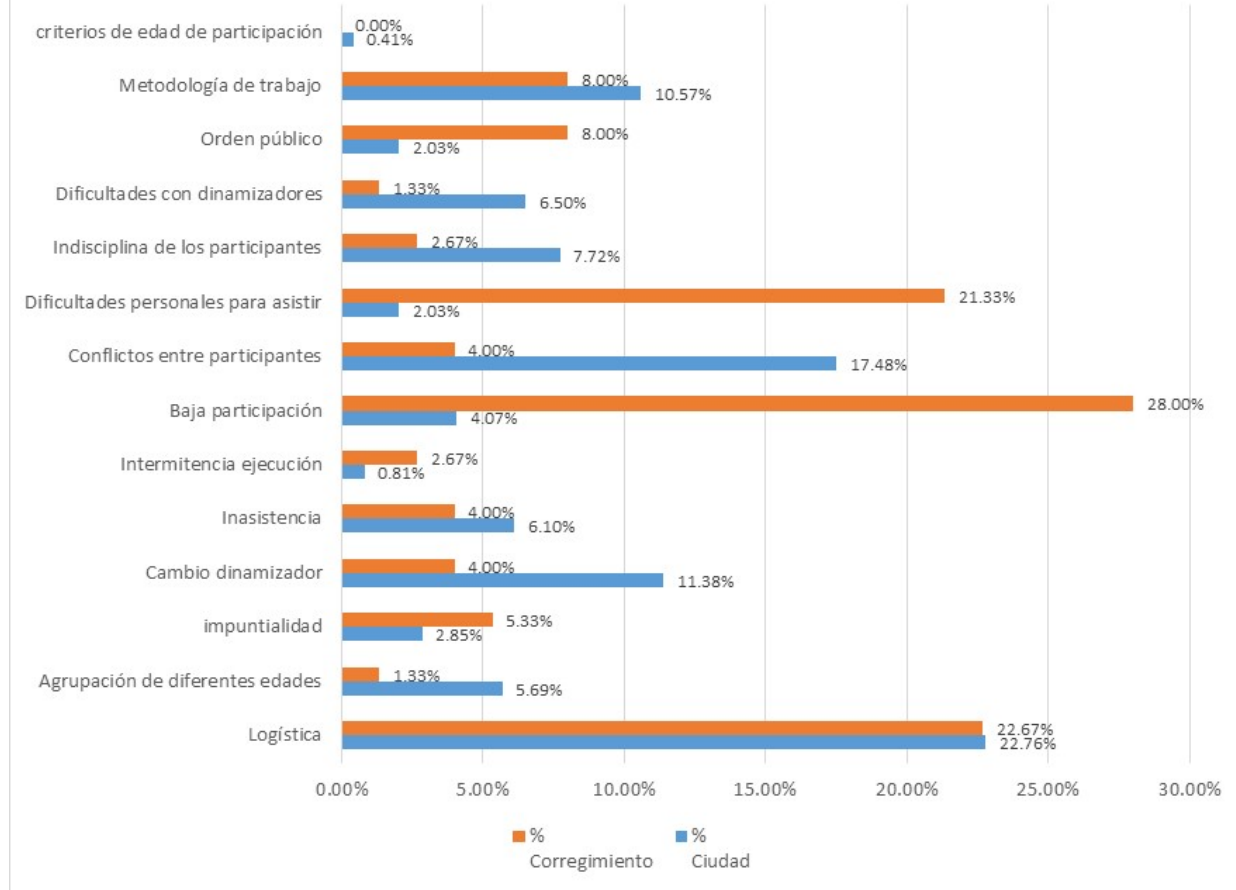

Fuente: elaboración propia

Ahora bien, con base a la información obtenida, puede decirse que las brechas presentadas no representan asuntos que sean trasversales al proceso de formación y promoción de la participación ciudadana, representando situaciones particulares que se presentaron en territorio específicos y que por tanto exige una atención particular para su mejora, por lo que no se encuentra en estos aspectos unas líneas de modificación significativa del proyecto en su concepción y diseño.

\section{DISCUSIÓN/ CONCLUSIONES}

A partir de la información levantada con los 321 entrevistados, es claro que en la ciudad de Medellín el proceso de formación y promoción de la participación que se viene desarrollando por varias décadas ha logrado impactar la vida de muchos niños, niñas y jóvenes de la ciudad, lo que ha posibilitado su integración a los territorios a partir de su reconocimiento y apropiación mediante actividades lúdico-pedagógicas, que les han abierto espacios de relacionamiento para la convivencia, construcción de valores, aprendizajes políticos, entre otros aspectos.

A partir de la experiencia de los entrevistados se ha evidenciado que los propósitos del proyecto se han cumplido de manera diferenciada, lo cual puede explicarse por la heterogeneidad de los participantes, tanto por el área de 
residencia -urbano y rural- como por las condiciones al interior de cada área, toda vez que en lo urbano se tienen comunas con condiciones sociales, culturales, económicas y políticas muy diferentes. Así mismo ocurre en lo rural, en donde los cinco corregimientos presentan condiciones que los hacen territorios muy diferentes entre sí. Siendo importante decir que este es uno de los componentes en el que se debe ganar mayor claridad en los participantes en vigencias posteriores, dado que si bien los aspectos reconocidos están vinculados al diseño del proyecto, muchos de ellos están en un nivel de contenido.

En el escenario deseado caracterizado por diferentes categorías desde el diseño del proyecto, se ha logrado un proceso en el cual la participación ciudadana va más allá de la participación orientada a mecanismos institucionales, integrando otras características de gran importancia, y que se asocia a las discusiones que en la materia se viene dando en Colombia y en otros países de América Latina, en donde se ha planteado la necesidad de promover en los procesos formativos y de participación modelos que integren la cotidianidad de la población de niños, niñas y jóvenes, desarrollando ejercicios que se basen en la vivencia directa y que sean prácticos e interactivos
(Martuccelly, 2016), integrando lenguajes propios de esta población que derivan en manifestaciones diferentes sobre la participación (Echavarría \& Carmona, 2017; Fernandez, 2014), Es claro que en este marco de discusión el proyecto logra asumir estos retos brindando espacios a los participantes a través de metodologías lúdico-pedagógicas que les permiten vincularse con contenidos asociados a la participación en los cuales la diversión, la recreación y la interacción vinculan sus intereses y responden a los aspectos que los motivan en esa etapa de la vida. En esto el proyecto ha logrado trascender los escenarios formales e institucionales de participación, lo cual ha sido de igual forma un reto en la participación de la población de niños, niñas y jóvenes (Arias \& Alvarado, 2015; Enríquez, 2015).

En este marco, el proyecto les ha permitido a los participantes generar relaciones con otros participantes del mismo barrio o vereda en marcos de tolerancia que motivan la interacción basada en el reconocimiento del otro, fortaleciendo la convivencia como eje central de la participación, respondiendo con ello a los retos actuales en materia de participación infantil y juvenil orientados a lograr relaciones horizontales que permitan la generación de consensos como base de la ciu- 
dadanía, fortaleciendo redes que trasciendan la acción individual (Vélez granada, 2014), fortaleciendo vinculación afectiva en escenarios de participación orientados a la construcción de paz y convivencia (Alvarado et al., 2006).

Otro aspecto que cobra gran relevancia en los resultados obtenidos es la vinculación al territorio como un factor estratégico en la dinamización de la participación ciudadana de la población participante, donde el reconocimiento de las dinámicas y asuntos del barrio o la vereda han sido aspectos que no solo han motivado a los niños, niñas y jóvenes, sino que además se han convertido en un eje de su acción formativa y de interacción. Encontrando aquí respuesta a un tercer reto en las discusiones en Colombia y América latina que tiene que ver con la necesidad de tomar en cuenta el contexto temporal y espacial de esta población (Quintero Mejía, Alvarado, \& Miranda, 2016), en donde "construir un marco de comprensión de las dinámicas infantiles y juveniles, objetivas y subjetivas, relacionadas con lo político y la ciudadanía, deben pasar a un plano más amplio y coherente con su realidad social y política, sus trayectorias, sus experiencias históricas y su cotidianidad"(Silva-nova, 2015. p. 285).
Un aspecto importante en el que debe avanzar el programa es en la vinculación de los participantes a los procesos comunitarios a través de actividades que les permita insertarse en los asuntos de interés de sus comunidades que son gestionados en las organizaciones sociales e institucionales, pues hasta ahora este aspecto aparece débilmente como una oportunidad, siendo estratégico para lograr uno de los propósitos centrales del proyecto que es la generación de liderazgos que permitan el relevo generacional futuro en los escenarios políticos de los territorios.

Finalmente, es importante decir que la formación y la promoción de la participación no puede ser asumida como una sola cosa o condición, los resultados muestran que la población apropia de manera muy diferente los contenidos que les entrega el programa, haciendo más relevantes unos elementos que otros, sin que ello signifique que no los trabaje, sino que es a través de unos que apropia los otros, y esto es relevante a la hora de entender los resultados de un proceso como estos. Las mejoras a este proyecto para la formación y promoción de la participación ciudadana deben orientarse a partir de aprovechar los aciertos evidenciados para tornarlos en oportunidades en experiencias futuras, dado 
que las brechas no evidenciaron aspectos que limiten significativamente el programa en su aplicación en toda la ciudad.

\section{REFERENCIAS BIBLIOGRÁFICAS}

Abad, M. (2002). La Relacion Entre Convivencia, Ciudadania Y Nueva. última Década, (16), 119-155

Alvarado, S., Ospina, H., Luna, M., \& Camargo, M. (2006). Transformación de actitudes frente a la equidad en niños y niñas de sectores de alta conflictividad social, en un proceso de socialización política y educación para la paz. Revista Latinoamericana de Ciencias Sociales, Niñez y Juventud, 4(1), 1-28.

Arias-Cardona, A. \& Alvarado, S. (2015). Youth and Politics: from formal participation to informal mobilization. Revista Latinoamericana de Ciencias Sociales, Niñez y Juventud, 13(2), 581594.

Benedicto, J. (2016). La ciudadanía juvenil: un enfoque basado en las experiencias vitales de los jóvenes. Revista Latinoamericana de Ciencias Sociales, Niñez y Juventud, 14(2), 925-938.

Consejo Municipal de Medellín. (2007). Acuerdo Municipal 043

Consejo Municipal de Medellín.

(2009). Acuerdo Municipal 083

Consejo Municipal de Medellín. (2010). Acuerdo Municipal 46

Carrasco, D., Carrasco, J. \& Guerra, E. (2014). Juventud y política en Antofagasta: Hacia una reinscripcion de la experiencia política en jóvenes. Última Década, (40), 85-107.
Corvera, N. (2014). Niñas y niños de Rosario y Montevideo: La voz de una nueva ciudadanía. Eure, 40(119), 193-216.

Echavarría, C. \& Carmona, D. (2017). Juventud , ciudadanía y posicionamientos políticos: una lectura desde el aula de clase *. Revista Latinoamericana de Estudios Educativos, 13(1), 153-178.

Enríquez, M. (2015). La ciudadanía intercultural en jovenes. Espacios Públicos, 18(44), 101-124.

Fernandez, A. (2014). Formación ciudadana: Jóvenes y acción social Citizenship Training: Youth and Social Action. Revista Electrónica de Investigación Educativa, 16, 29-42.

Gülgönen, T. (2016). Participación infantil a nivel legal e institucional en México¿Ciudadanos y ciudadanas?. Revista Latinoamericana de Ciencias Sociales, Niñez y Juventud, 14(1), 81-93.

Gutierres, M. \& Morales, J. (2015). Formación de Ciudadanos: Conceptualización y Desarrollo de la competencia social y cívica. Educación XXI, 18(1), 259-282.

Kriger, M., \& Daiban, C. (2011). Del ideal del ciudadano al ciudadano en-situación: un estudio sobre los modelos de ciudadanía y los posicionamientos subjetivos de jóvenes ciudadanos en la Argentina actual ( Buenos Aires y Conurbano, 2011-13 ). Folios, (41), 87-102.

Martínez, M., Silva, C., Morandé, M., \& Canales, L. (2010). Los jóvenes ciudadanos: reflexiones para una política de formación ciudadana juvenil. Ultima Década, 18(32), 105-118.

Martuccelly, D. (2016). Condición Adolescente y Ciudadanía Escolar. Educação \& Realidade, 41(1), 155-174. 
Ñáñez, J., \& Castro, H. (2016). Educación artística y formación ciudadana: espacio para forjar la sensibilidad en la Corporación Colegio San Bonifacio, de Ibagué, Colombia. Entramado., 12(2), 154-165.

Osorio, A. (1986). La ampliación de la participación ciudadana infantil en México. Una aproximación sociológica a sus razones, obstáculos y condiciones. Sociológica, 31(87), 111-142.

Quintero, M., Alvarado, S., \& Miranda, J. (2016). Conflicto armado, variables socioeconómicas y formación ciudadana: un análisis de impacto. Revista Latinoamericana de Ciencias Sociales, niñez y juventud, 14(1), 147-161.

Reguillo, R. (2003). Ciudadanías juveniles en América Latina. Última Década, (19), 11-30.

Silva-nova, S. (2015). Citizenship and youth: a brief review. Diversitas: Perspectivas en Psicología, 17(2), 273-288.

Vélez granada, J. F. (2014). La construccion de ciudadania: institucionalidad y juventud. Última Década, (19), 1-10.

Universidad de Antioquia., Alcaldía de Medellín (2013). Convenio Interadministrativo para la creación y consolidación de Semilleros de Participación. Medellín.

\section{PARA CITAR ESTE ARTÍCULO:}

Brand Monsalve, E. (2018). Análisis de aciertos y brechas en la promoción de la participación ciudadana en niños, niñas y jóvenes de la ciudad de medellín. Caso proyecto semilleros infantiles para la participación. Collectivus, Revista de Ciencias Sociales, 5(2), 130-152.

DOI: http://dx.doi.org/10.15648/Coll.2.2018.5

Recibido: 27/03/2018

Aprobado: 02/06/2018 